
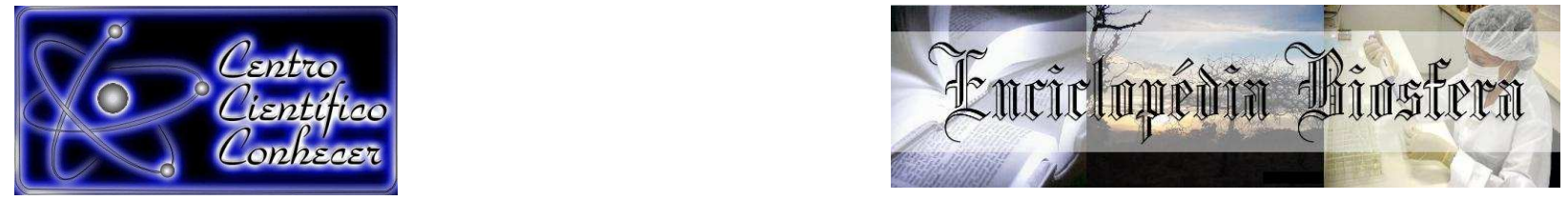

\title{
LEVANTAMENTO PRELIMINAR DA ANÁLISE PARASITÁRIA DE SUÍNOS ADAPTADOS LOCALMENTE NA REGIÃO CENTRO-SUL DO ESTADO DO PARANÁ-BRASIL
}

Maria Marta Loddi ${ }^{*}$; Denyse Maria Galvão Leite ${ }^{2}$; Raquel Abdallah da Rocha ${ }^{1}$, Ana Cláudia Andrzejeski ${ }^{3}$, Juliana Stocco Martins ${ }^{1}$.

${ }^{1}$ Universidade Estadual de Ponta Grossa, Departamento de Zootecnia, Ponta Grossa - Paraná - Brasil.

${ }^{2}$ Instituto Agronômico do Paraná, Pesquisadora na Área de Zootecnia, Ponta Grossa - Paraná - Brasil.

${ }^{3}$ Centro de Ensino Superior dos Campos Gerais, Ponta Grossa - Paraná - Brasil. Ioddimm@yahoo.com.br

Recebido em: 08/09/2015 - Aprovado em: 14/11/2015 - Publicado em: 01/12/2015 DOI: http://dx.doi.org/10.18677/Enciclopedia_Biosfera_2015_184

O presente trabalho teve como objetivo realizar um levantamento preliminar parasitológico em uma criação de suínos localmente adaptados, oriundos de uma comunidade tradicional conhecida como Faxinal, localizada na região Centro-Sul do Estado do Paraná, Brasil. Foram obtidas 37 amostras de fezes de suínos, machos e fêmeas, com idade entre seis meses à seis anos. $O$ delineamento experimental foi inteiramente casualizado, distribuído em dois fenótipos de raça: 18 Fenótipo com características Moura e 19 animais de outros tipos de fenótipos (Caruncho, Canastra, Nilo de brinco, Cuié e Casco de Burro. Amostras de fezes para determinação do número de ovos por grama de fezes (OPG) foram colhidas diretamente da ampola retal. Os suínos do fenótipo Moura apresentaram maiores médias de OPG para Strongyloides spp. e Ascaris spp. $(\mathrm{P}<0,05)$. Observaram-se as seguintes prevalências: $91,89 \%$ para nematoides gastrintestinais (34 animais positivos/37 animais totais), 29,73\% para Strongyloides spp. (11 animais positivos/37 animais totais), $18,92 \%$ para Ascaris spp. ( 7 animais positivos/37 animais totais), além de dois animais positivos para Coccídeo $(5,4 \%)$. No exame quantitativo (mínimo e máximo), obteve-se: 0-12.250 OPG para ovos do tipo Estrongilídeos; 0250 OPG para Strongyloides spp.; 0-250 OPG para Ascaris spp. Com os resultados obtidos será possível implantar um programa de manejo sanitário adequado as condições ambientais do Faxinal, respeitando a rotina de criação dos suínos por esta comunidade tradicional. Evidencia-se, com isto, a importância do manejo tecnificado no controle parasitário de suínos localmente adaptados.

PALAVRAS- CHAVE: comunidade tradicional, OPG, sanidade animal, suínos extensivos 


\title{
SURVEY PRELIMINARY PARASITE SWINE ADAPTED LOCALLY ANALYSIS IN THE REGION CENTRAL SOUTH-BRAZIL PARANA STATE
}

\begin{abstract}
This study aimed to carry out a parasitological preliminary survey in a pig breeding locally adapted, coming from a traditional community known as Faxinal, located in the South Central region of Parana State, Brazil. They were obtained 37 samples of swine feces, male and female, aged six months to six years. The experimental design was completely randomized, distributed in two race phenotypes: 18 animals from Phenotype with Moura features and 19 animals from other phenotypes (Caruncho, Canastra, Nilo de brinco, Cuié e Casco de Burro). Feces samples were collected directly from the rectum to determine the number of eggs per gram of feces (EPG). Pigs of Moura phenotype had higher averages OPG to Strongyloides spp. and Ascaris spp. ( $P<0.05)$. The following prevalences were observed: $91.89 \%$ for gastrointestinal nematodes (34 positive animals / 37 total animals), $29.73 \%$ for Strongyloides spp. (11 positive animals / 37 total animals), 18.92\% for Ascaris spp. (7 positive animals / 37 total animals), and two positive animals coccidia (5.4\%). On quantitative examination (minimum and maximum), there were obtained: $0-12,250$ OPG for eggs for the strongyles type; 0-250 OPG to Strongyloides spp.; 0-250 OPG to Ascaris spp. With this results will be possible deploy a health management program for suitable environmental conditions of Faxinal, respecting the routine raising pigs for this traditional community. It is evident, thus, the importance of technical management in parasite control of pigs locally adapted.
\end{abstract}

KEYWORDS: traditional community, EPG, animal health, extensive swine

\section{INTRODUÇÃO}

Os faxinais são comunidades tradicionais definidas como territórios sociais típicos da região centro-sul do estado do Paraná. Sua formação data do início do século $X X$, e sua organização está baseada no uso comum da terra em sistema silvipastoril. Segundo CHANG (1988), este modo de uso da terra, de forma genérica, pode ser dividido em dois espaços separados por cercas: as "terras de criar" ou "criadouro comunitário" de uso comum dos moradores onde se encontram suas casas, seus animais e a floresta; e as "terras de plantar", que se constituem em áreas de uso particular de cada morador, onde se desenvolve a agricultura de subsistência.

No sistema Faxinal a criação de suínos localmente adaptados ou de raças naturalizadas/locais/crioulas foi por muito tempo uma das principais atividades, a qual tinha grande importância socioeconômica por promover ingresso e possibilidade de trabalho, assim como, de renda para as famílias. Com a modernização da agricultura, bem como a demanda por animais mais produtivos e com menor teor de gordura na carcaça, os suínos naturalizados foram desvalorizando no mercado levando muitos produtores a deixarem a atividade ou reduzirem seus rebanhos ou substituírem as raças locais por raças exóticas. Apesar das mudanças ocorridas nos Faxinais, estudos realizados, nos últimos 10 anos, têm verificado que a criação de suínos localmente adaptados é uma atividade representativa destas comunidades (LEITE et al., 2012; LEITE \& LODDI (2012). A produção é destinada principalmente para o consumo familiar e o excedente a comercialização (LEITE et al., 2009). 
LEITE et al. (2009) verificaram que a maioria dos produtores do Faxinal Taquari dos Ribeiro, município de Rio Azul - PR, comercializam suínos $(40,74 \%)$, mas também bovinos $(22,22 \%)$, ovinos $(14,81 \%)$, caprinos $(7,41 \%)$, aves $(7,41 \%)$ e abelhas $(7,41 \%)$. LEITE \& LODDI (2012) verificaram que o tamanho médio dos rebanhos de suínos nos Faxinais Kurger e Marcondes, localizados na região CentroSul do Estado do Paraná-Brasil, eram de 18,17 e 6,12 cabeças, respectivamente. Foi também observado que a maioria dos rebanhos eram constituídos por animais mestiços sem raça definida, provavelmente, remanescentes das raças naturalizadas de suínos.

Os suínos localmente adaptados no Brasil são conhecidos pela rusticidade, como resistência às doenças e ectoparasitas, boas qualidades maternas e adaptadas às condições de manejos precários por terem uma conformação corporal para deposição de gordura e por serem menos exigentes em manejo se comparados às raças suínas melhoradas (MARIANTE et al., 2003). De acordo com MARIANTE et al. (2003), os suínos locais também são capazes de sobreviver em diferentes ecossistemas das regiões brasileiras mesmo em condições desfavoráveis de criação. Em geral, como acontece na maioria dos países, as raças suínas locais são encontradas apenas em pequenos criatórios que estão distribuídos nas diferentes regiões do País (SILVA et al., 2011).

Quanto à situação das raças suínas localmente adaptadas no Brasil, das 13 raças e/ou ecótipos identificados apenas duas foram registradas na Associação Brasileira de Criadores de Suínos: a raça Piau e a raça Moura. A raça Moura foi originada do cruzamento entre as raças localmente adaptadas no Brasil, como Canastrão e Canastra e a raça exótica Duroc (EGITO et al., 2002). Essa raça é considerada uma das mais antigas e preservadas até hoje. As características marcantes para criação da raça Moura são a sua rusticidade, prolificidade, o comprimento de carcaça e o marmoreio na carne. Os animais possuem pelagem preta entremeada de pelos brancos, orelhas do tipo ibéricas grandes e firmes e perfil fronto-nasal retilíneo a subcôncavilíneo (EGITO et al., 2002). Segundo FÁVERO et al. (2007) a raça Moura apresenta índices produtivos inferiores às raças exóticas, porém pode ser utilizada para produção de presunto curado.

A prática do pastoreio contínuo dos animais e as condições rústicas de nutrição, higiene e sanidade comuns nos Faxinais podem favorecer a incidência de verminose nos rebanhos. No entanto, são poucas as informações na literatura a respeito desse tema. Postula-se que a ocorrência de doenças parasitárias entéricas pode interferir na produtividade do rebanho, portanto, o diagnóstico do grau de infecção animal por meio de exames clínicos e laboratoriais e o conhecimento da epidemiologia dos parasitos são indispensáveis na formulação de um programa de controle sanitário eficiente. Assim, o presente trabalho teve como objetivo realizar um levantamento preliminar das infecções por parasitas em uma criação de suínos localmente adaptados, oriundos de uma comunidade tradicional conhecida como Faxinal, localizada na região Centro-Sul do estado do Paraná, Brasil.

\section{MATERIAL E MÉTODOS}

\section{Local do experimento}

A parte de campo do experimento foi realizada em um Faxinal, localizado na cidade de São Mateus do Sul, na região Centro-Sul do estado do Paraná, Brasil. Já 
a parte laboratorial foi realizada no Laboratório de Parasitologia do Departamento de Zootecnia, Universidade Estadual de Ponta Grossa, Paraná, Brasil.

\section{Descrição do experimento}

Foram utilizados 37 suínos localmente adaptados, machos e fêmeas, com idade entre seis meses à seis anos. O delineamento experimental utilizado foi inteiramente casualizado, distribuído em dois fenótipos: Fenótipo com características Moura e outros tipos de fenótipos (Caruncho, Canastra, Nilo de brinco, Cuié e Casco de Burro), sendo 18 animais do primeiro fenótipo (Moura) e 19 animais dos outros fenótipos citados.

\section{Exame parasitológico}

A coleta de fezes foi realizada no mês de abril de 2015. O exame parasitológico foi realizado segundo metodologia de GORDON \& WHITLOCK (1939) para contagem de ovos por grama de fezes (OPG). As amostras fecais foram colhidas diretamente da ampola retal, utilizando-se sacos plásticos previamente identificados. Posteriormente as amostras foram mantidas caixas isotérmicas contendo gelo reciclável, e transportadas ao Laboratório de Parasitologia - UEPG, quando em seguida eram processadas.

\section{Cálculo da prevalência}

A prevalência foi obtida a partir do número de indivíduos que foram positivos para cada parasita examinado dividido pelo número total de indivíduos da população estudada, em porcentagem.

\section{Análise estatística}

Os dados referentes aos valores de OPG foram submetidos à análise de variância com a utilização do programa Minitab ${ }^{\circledR}$ (Versão 17). Para tal, os mesmos foram previamente transformados $(\log (x+1))$. No entanto, para facilitar a interpretação dos resultados, as médias aritméticas é que serão apresentadas. As médias foram comparadas pelo Teste de $t$ de Student, e consideradas significativas quando $\mathrm{P}<0,05$.

\section{RESULTADOS E DISCUSSÃO}

O protocolo experimental foi aprovado pela Comissão de Ética do Uso de Animais (CEUA), pelo processo de n. 23/2014 da CEUA/IAPAR.

Foi verificado maior número de ovos de Strongyloides spp. $(\mathrm{P}<0,06)$ para os animais do fenótipo Moura em relação aos outros fenótipos de suínos encontrados no Faxinal (Tabela 1). Isso pode ter-se dado em função de que os animais do fenótipo Moura foram introduzidos em até três meses anterior à data da coleta. Assim, os animais dos outros fenótipos, com maior tempo de permanência no Faxinal, indicam que são mais adaptados ao ambiente, como preconizado por MARIANTE \& CAVALCANTE (2006) onde concluíram que as raças suínas localmente adaptadas no Brasil são conhecidas por possuírem características importantes, como resistência às doenças, boas qualidades maternas e adaptadas às condições de manejos precários. Os mesmos resultados foram encontrados para a contagem de Ascaris spp., visto que se observou um maior número de OPG para 
os animais do fenótipo Moura $(\mathrm{P}<0,05)$. Não foi verificada diferença significativa para o número de ovos de nematoides gastrintestinais entre os fenótipos estudos.

TABELA 1: Valores médios (desvio-padrão) da contagem de OPG (ovos por grama de fezes) de nematoides gastrintestinais, Strongyloides spp. e Ascaris spp. de suínos criados no sistema Faxinal na região Centro-Sul do Estado do Paraná, Brasil.

\begin{tabular}{l|c|c}
\hline \multirow{2}{*}{ Parasitas } & \multicolumn{2}{|c}{ Fenótipo } \\
\cline { 2 - 3 } & Moura & Outros \\
\hline Nematoides gastrintestinais & $1972(3512)$ & $1284(1886)$ \\
Strongyloides spp. & $64(90,4)^{\star}$ & $18(44,8)^{\star}$ \\
Ascaris spp. & $50(95,5)^{\star *}$ & $3(11,47)^{\star *}$ \\
\hline
\end{tabular}

*na linha, indica diferença significativa, $\mathrm{P}<0,06$;

**na linha, indicam diferença significativa, $P<0,05$.

Observou-se prevalência de 91,89\% para os estrongilídeos (Tabela 2). Em Sergipe, BRITO et al. (2012) também encontraram altas prevalências de nematódeos gastrintestinais (estrongilídeos) em suínos criados extensivamente. D'ALENCAR et al. (2011) analisaram a influência do manejo higiênico-sanitário nos níveis de infecção por parasitas gastrintestinais em suínos de granjas tecnificadas e de subsistência abatidos na Região Metropolitana de Recife e Zona da Mata do estado de Pernambuco, Brasil, e observaram prevalência de 2,5\% para estrongilídeos, achados estes 97\% inferior aos encontrados no presente experimento. Já KÚ et al. (2013) verificaram a prevalência de parasitas gastrintestinais em suínos de raças locais do México e criados extensivamente e a prevalência dos parasitas encontrados foi de 71,9; 4,7; 6,3 e 3,1\% para Oesophagostomum spp., Strongyloides spp., Trichuris suis, Ascaris suun, respectivamente.

TABELA 2. Taxa de prevalência dos parasitas encontrados em suínos criados no sistema Faxinal na região Sul do Estado do Paraná, Brasil.

\begin{tabular}{lc}
\hline \multicolumn{1}{c}{ Parasita } & Prevalência (\%) \\
\hline Nematoides gastrintestinais & $91,89(34 / 37)$ \\
Ascaris spp. & $18,92(7 / 37)$ \\
Strongyloide spp. & $29,73(11 / 37)$ \\
Coccídeo & $5,40(2 / 37)$ \\
\hline
\end{tabular}

Os nematoides gastrintestinais dos suínos criados em sistemas extensivos apresentam importância do ponto de vista de saúde animal e econômico. Kú et al. (2013) verificaram que níveis que infecções maiores de 400 OPG para nematoides intestinais estavam correlacionados com a redução de desempenho produtivo de suínos criados extensivamente. Suínos criados a pastos geralmente estão parasitados por Hyostrongylus rubidus, parasita do estômago e Oesophagostomum spp., parasita do intestino grosso (MONTEIRO, 2014). Hyostrongylus rubidus é um parasita hematófago e mesmo em infecções inaparentes, determina redução no ganho de peso e na conversão alimentar. Já os nódulos formados na mucosa do intestino grosso, em resposta à penetração das larvas de Oesophagostomum spp., prejudicam a absorção de nutrientes e a motilidade intestinal (MONTEIRO, 2014). 
No presente experimento não se realizou coproculturas, pois as quantidades de fezes colhidas dos animais foram insuficientes para tal.

Ascaris spp. também foi encontrado nos exames de fezes realizados com prevalência de 18,92\% (Tabela 2). LIMA et al. (2014) determinaram a ocorrência de helmintos gastrintestinais de suínos. Verificaram maior prevalência de Ascaris spp. diferindo dos achados do presente estudo. Ascaris suum provoca grandes perdas de produção em suínos devido à redução do crescimento, déficit de crescimento e mortalidade (JEX et al. 2011). Ao exame quantitativo (mínimo e máximo), obteve-se: 0-250 OPG para Ascaris spp.

Em relação à Strongyloides spp., 29,73\% dos animais estavam parasitados com este nematoide (Tabela 2). Strongyloides spp. atingem principalmente animais jovens, podendo ainda afetar fêmeas em lactação. Como consequência do parasitismo, os animais apresentam diarreia, má absorção dos nutrientes e desidratação, acarretando em atraso no desenvolvimento podendo levar à morte (MONTEIRO, 2014). No presente experimento, dos 11 animais que apresentaram este parasita, oito eram com idade inferior à 24 meses. Ao exame quantitativo (mínimo e máximo), obteve-se: 0-250 OPG para Strongyloides spp.

$E$ ainda, foram encontrados nos exames parasitológicos dois animais positivos para coccídeo $(5,4 \%)$. A coccidiose suína, causada pelo Isospora suis, se destaca entre as doenças entéricas mais frequentes na produção suinícola (CAMPOS et al., 2012). Caracteriza-se por uma enterite grave com consequente diarreia em leitões de uma a duas semanas de idade (MONTEIRO, 2014).

LEITE et al. (2013) avaliaram exames coprológicos das 203 amostras de fezes dos animais criados no Faxinal Taquari dos Ribeiro, município de Rio Azul PR. Observaram 87,5\%, 95,31\%, 100\% e 100\%, de incidência de verminose nos rebanhos de bovinos, ovinos, caprinos e suínos, respectivamente. Neste estudo verificou-se que $86,4 \%$ dos animais estudados apresentaram algum tipo dos parasitas analisados, independente da carga parasitária.

Os resultados do presente estudo mostram que os animais criados neste sistema apresentaram relativas contagens de OPG. Portanto, verifica-se a necessidade do estabelecimento de práticas de manejo sanitário, específicas para os Faxinais, que possam minimizar a prevalência de verminoses nos suínos localmente adaptados.

\section{CONCLUSÃO}

Com os resultados obtidos será possível implantar um programa de manejo sanitário adequado às condições ambientais do Faxinal, respeitando a rotina de criação dos suínos por esta comunidade tradicional. Neste sistema de criação, os suínos caracterizados com fenótipo da raça Moura apresentaram-se mais susceptíveis às infecções por nematoides gastrintestinais comparado com os outros fenótipos estudados.

\section{REFERÊNCIAS}

BRITO, G. G.; SANTOS, T. B.; MELO, C. M.; JERALDO, V. L. S. Ocorrência de enteroparasitas em amostras fecais de suínos do município de Simão Dias - SE. Cadernos de Graduação - Ciências Biológicas e da Saúde. v. 1 n.15, p. 11-18, 2012. 
CAMPOS, T. M.; CARVALHO, T. A.; CARVALHO, G. D.; PINTO, R.; MOLINO, J. P.; SOUZA, M. R. Coccidiose suína. Revista Eletrônica Nutritime. v. 9, n. 2, p. 17261739, 2012.

CHANG, M.Y. Sistema faxinal: uma forma de organização camponesa em desagregação no centro-sul do Paraná. Londrina: IAPAR, 1988. (Boletim Técnico).

D'ALENCAR, S.; FARIAS, M. P. O.; ROSAS, E. O.; LIMA, M. M.; ALVES, L. C.; FAUSTINO, M. G. A. Influência do manejo higiênico-sanitário na infecção por helmintos gastrintestinais em suínos de granjas tecnificadas e de subsistência abatidos na região metropolitana de recife e zona da mata do Estado de Pernambuco, Brasil. Arquivo do Instituto Biológico. v. 78, n. 2, p. 207-215, 2011.

EGITO, A. A.; MARIANTE, A. S.; ALBUQUERQUE, M. S. M. Programa Brasileiro de Conservação de Recursos Genéticos Animais. Archivos de Zootecnia , v. 51, p. 3952, 2002.

FAVERO, J. A.; FIGUEIREDO, E. P.; FEDALTO, L. M.; WOLOSZN, N. A raça de suínos moura como alternativa para a produção agroecológica de carne. Revista Brasileira de Agroecologia, v. 2, n.1, 2007.

GORDON, H. M. C. L.; WHITLOCK, H.V. A new technique for counting nematode eggs in sheep faeces. Journal Council Science Industry Research. v. 12, n.1, p. 103-112, 1939.

JEX, A. R.; LIU, S.; LI, B.; YOUNG, N. D.; HALL, R. S.; LI, Y.; YANG, L.; ZENG, N.; XU, X.; XIONG, Z.; CHEN, F.; WU, X.; ZHANG, G.; FANG, X.; KANG, Y.; ANDERSON, G. A.; HARRIS, T. W.; CAMPBELL, B. E.; VLAMINCK, J.; WANG, T.; CANTACESSI, C.; SHWARZ, E. M.; RANGANATHAN, S.; GELDHOF, P.; NEJSUM, P.; STERNBERG, P. W.; YANG, H.; WANG, J.; WANG, J.; GASSER, R. B. Ascaris suum draft genome. Nature. V. 479, p. 529-536, 2011.

KÚ, R.; TREJO, W.; AGUILAR, A.; BELMAR, R.; CASTILHO, J. Parasitismo gastrointestinal en el cerdo pelón mexicano en traspatio en el estado de Yucatán, México. Revista Colombiana de Ciencia Animal, v. 6, n.1, 2013.

LEITE, D. M. G., CHERUBIM, A. A.; PEREIRA, J. M. A. Caracterização da Criação Animal em Sistema Faxinal. Revista Brasileira de Agroecologia, v. 4, n.2, 2009.

LEITE, D. M. G.; LODDI, M. M. Caracterização dos Rebanhos de Suínos em Sistema Faxinal. Actas Iberoamericans de Conservacion Animal, v. 2, p. 115-117, 2012

LEITE, D. M. G.; LODDI, M. M.; CHERUBIM, A. A. Caracterização do rebanho suíno no 'Sistema Faxinal Marmeleiro de Baixo'. In: IX Congresso da Sociedade Brasileira de Sistemas de Produção (IX CSBSP) - Ciência, tecnologia e inovação para o desenvolvimento rural sustentável, 2012, Luziânia. IX Congresso da Sociedade Brasileira de Sistemas de Produção (IX CSBSP) - Ciência, tecnologia e inovação para o desenvolvimento rural sustentável. Planaltina: Embrapa Cerrados, 2012. CD ROM 
LEITE, D. M. G.; CHERUBIM, A. A.; LODDI, M. M. Faxinal Taquari dos Ribeiros: diagnóstico de verminose nos rebanhos. Cadernos de Agroecologia, v.8, n.2, 2013

LIMA, D. S.; PORTO, A. G.; CARVALHO, C. D.; RODRIGUES, S. A.; MELO, C.; M.; JERALDO, V. L. S. Parasitas gastrointestinais de suínos: prevalência de infecção e controle com utilização do alho (Allium sativum L.). Sempesq, n. 16, 2014.

MARIANTE, A. S.; CASTRO, S. T. R.; ALBUQUERQUE, M. do S. M.; PAIVA, S. R.; GERMANO, J. L. Pig biodiversity in Brazil. Archivos de Zootecnia, v. 52, p. 245248, 2003.

MARIANTE, A. S.; CAVALCANTI, N. Animais do descobrimento: raças domesticas da história do Brasil. Brasília: Embrapa Sede/Embrapa Recursos Genéticos e Biotecnologia. 2006. 274p.

MONTEIRO, S. G. Parasitologia na Medicina Veterinária. 1 ed, Roca, 356p, 2014.

SILVA, E. C.; DUTRA JUNIOR, W. M.; IANELLA, P.; GOMES FILHO, M. A.; OLIVEIRA, J. P.; FERREIRA, D. N. M.; CAETANO, A. R.; PAIVA, S.R. Patterns of genetic diversity of local pig populations in the State of Pernambuco, Brazil. Revista Brasileira de Zootecnia, v.40, n. 8, p.1691-1699, 2011. 